\title{
Predictors of Loss to Follow-Up among Men with Tuberculosis in Puducherry and Tamil Nadu, India
}

\author{
Thomas J. Zhou, ${ }^{1} \dagger$ Subitha Lakshminarayanan, ${ }^{2}$ † Sonali Sarkar, ${ }^{2}$ Selby Knudsen, ${ }^{3}$ C. Robert Horsburgh Jr. ${ }^{1,3,4}$ \\ Muthuraj Muthaiah, ${ }^{5}$ Carolyn K. Kan, ${ }^{6}$ Padmini Salgame, ${ }^{7}$ Jerrold J. Ellner, ${ }^{7}$ Gautam Roy, ${ }^{2}$ Helen E. Jenkins, ${ }^{1}$ and \\ Natasha S. Hochberg ${ }^{3,4 *}$ \\ ${ }^{1}$ Department of Biostatistics, Boston University School of Public Health, Boston, Massachusetts; ${ }^{2}$ Department of Preventive and Social Medicine, \\ Jawaharlal Institute of Postgraduate Medical Education and Research, Puducherry, India; ${ }^{3}$ Department of Medicine, Section of Infectious \\ Diseases, Boston University School of Medicine, Boston, Massachusetts; ${ }^{4}$ Department of Epidemiology, Boston University School of Public \\ Health, Boston, Massachusetts; ${ }^{5}$ Intermediate Reference Laboratory, Government Hospital for Chest Diseases, Puducherry, India; ${ }^{6}$ Department of \\ Medicine, Boston University School of Medicine, Boston, Massachusetts; ${ }^{7}$ Department of Medicine, Rutgers New Jersey Medical School, Newark, \\ New Jersey
}

\begin{abstract}
Identifying predictors of loss to follow-up (LTFU; treatment lapse $\geq 2$ months) among people with tuberculosis (TB) may assist programmatic efforts in controlling the spread of TB. Newly diagnosed smear-positive TB patients were enrolled in the Regional Prospective Observational Research for TB study in Puducherry and Tamil Nadu, India. Treatment records were used to identify LTFU of those who were enrolled from May 2014 through December 2017. This nested case-control study evaluated male TB patients. Predictors were assessed using multivariable logistic regression. Of 425 men with TB, 82 (19\%) were LTFU. In the adjusted analyses of males, divorced/separated marital status (adjusted odds ratio [aOR] 3.80; 95\% Cl: 1.39-10.38) and at-risk alcohol use (aOR 1.92; 95\% Cl: 1.12-3.27) were significant predictors for increased risk of LTFU, and diabetes was a significant predictor for decreased risk of LTFU (aOR 0.52 ; 95\% $\mathrm{Cl}$ : 0.29-0.92). Of 53 men with recorded date of last treatment visit, $23(43 \%)$ and $43(81 \%)$ had LTFU within the first 2 and first 4 months of treatment, respectively. Addressing at-risk alcohol use and providing more intensive follow-up could lead to improved treatment completion.
\end{abstract}

\section{INTRODUCTION}

Tuberculosis (TB) is the leading infectious cause of death worldwide, with more than 1.6 million TB deaths in $2018 .{ }^{1}$ In the same year, India accounted for $27 \%$ of the 10.0 million incident cases worldwide. ${ }^{1}$ The Indian government's Revised National Tuberculosis Control Programme (RNTCP), now renamed as the National Tuberculosis Elimination Program, provides public sector TB diagnosis and treatment. In 2016, RNTCP reported that $74 \%$ of TB patients were cured. ${ }^{2}$ Loss to follow-up (LTFU) and ineffective treatment may result in poor outcomes and increased transmission. ${ }^{3}$

The reported LTFU (treatment interruption for $\geq 2$ consecutive months) rates varied among different Indian states, and ranged between $1 \%$ and $6 \%$ and $2 \%$ and $15 \%$ in newly and previously treated patients, respectively. ${ }^{2}$ Patients with LTFU (previously referred to as defaulted) often have poorer treatment outcomes, higher risk of relapse, and are more prone to developing drug resistance. $^{4-7}$ Improving treatment completion and reducing LTFU is a key step toward TB control in India.

Predictors of LTFU in India are likely to be region specific. Although LTFU rates in southern India have been reported to range from $7.4 \%$ to $20 \%,{ }^{8}$ only one study assessed multivariable models of LTFU predictors within this region, and those data are from almost 20 years ago. ${ }^{9}$ With programmatic efforts to reduce LTFU in India, it is likely that risk factors have changed during this time, thus prompting another study to revisit this issue. Factors such as gender, alcoholism, provider-patient interactions, distance to treatment center, and anti-TB drug side effects were reported from studies in northern and eastern

*Address correspondence to Natasha S. Hochberg, Department of Medicine, Section of Infectious Diseases, Boston University School of Medicine, 801 Massachusetts Ave., Crosstown 2012, Boston, MA 02118. E-mail: nhoch@bu.edu

†These authors contributed equally to this work.
India, ${ }^{10-12}$ whereas inadequate TB knowledge and illiteracy were highlighted as factors in central India. ${ }^{13}$

This nested case-control study aimed to identify LTFU predictors for newly diagnosed pulmonary TB patients in southern India, specifically Puducherry and Tamil Nadu. We sought to identify whether socioeconomic characteristics (e.g., education and poverty), behavioral characteristics (e.g., smoking and alcohol use, and household alcohol use), comorbidities, lack of knowledge about TB, or external factors (season) may be predictors of LTFU in this region. The analyses are restricted to male TB patients (because nearly all LTFU occurred in males) and their household contacts.

\section{MATERIALS AND METHODS}

Study population and design. Investigators at the Jawaharlal Institute of Postgraduate Medical Education and Research (JIPMER), Boston Medical Center (BMC), and Rutgers University conducted this observational household contact cohort study as part of the Regional Prospective Observational Research for TB (RePORT)-India. Methods have been described in detail previously. ${ }^{14-17}$ In brief, participants were identified through RNTCP clinics in Puducherry and the Tamil Nadu districts of Villupuram and Cuddalore. Individuals suspected of having TB seek care from public and private providers, but those in the public system ultimately undergo sputum testing at government laboratories; those with evidence of TB are referred to primary healthcare centers for treatment through directly observed therapy, short course (DOTS). Adherence is assessed through treatment cards by DOTS providers. Newly diagnosed pulmonary TB cases (category I), whose sputum was $\geq 1$ Ziehl-Neelsen stain positive for acid-fast bacilli and culture positive for Mycobacterium tuberculosis, were enrolled based on the inclusion criteria of $\geq 6$ years of age (as younger individuals are candidates for latent TB infection treatment through RNTCP), no TB treatment 
history, and intention to complete treatment under DOTS. The exclusion criteria were having multidrug or extensively drugresistant TB (as the aim of RePORT was to identify predictors of treatment failure for drug-sensitive TB) or being extremely ill (Karnofsky score $\leq 10$ ).

This is a nested case-control study within the RePORT cohort, where cases were selected as those with LTFU and controls as those who were not. Data were collected for all patients who were enrolled from May 2014 through December 2017. We then restricted the data to only those who had a final treatment outcome. Participants were treated by RNTCP according to national guidelines. ${ }^{18}$ Household contacts were also enrolled and interviewed. All participants were interviewed at a mutually acceptable location (usually the participant household) and interviewed in Tamil by local field staff.

Data collection. Study personnel administered questionnaires that addressed demographic, socioeconomic, and clinical characteristics, including age, gender, caste, municipality, education, household income, knowledge about TB, TB history, substance use, and presence of comorbidities. Those with HIV infection were referred to the state HIV treatment program, as per RNTCP guidelines; those found to have other comorbidities were instructed to discuss these with the medical officer at the primary healthcare center. Visits at 2 months addressed symptoms. Sputum smear and culture results were obtained from RNTCP with repeat smear and culture confirmation performed by study personnel at enrollment. Data from RNTCP on 2-month and end-of-treatment smear and culture were obtained when available. Scanned copies of questionnaires were transferred to BMC using Verity TeleForm version 10.8 (Sunnyvale, CA) and read into a Microsoft Access (Seattle, WA) database.

Measurements and definitions. The RNCTP DOTS records and treatment completion information were reviewed to identify LTFU. For subjects with RNTCP treatment completion information, the final outcome was determined based on RNTCP classification as LTFU. For those missing RNTCP treatment outcomes, LTFU was identified by study staff if they had not completed treatment and were no longer able to be contacted (up to three attempts). For those with DOTS records, LTFU date was defined as the last recorded DOTS dose before a lapse of $\geq 2$ months. The Alcohol Use Disorders Identification Test (AUDIT-C), scored from 0 to 12, identified at-risk drinkers (score $\geq 4$ for males and $\geq 3$ for females) for participants and their household contacts. ${ }^{19}$ The Household Food Insecurity Access questionnaire identified those with food security, and mild, moderate, and severe food insecurity. ${ }^{20}$ The Multidimensional Poverty Index (MPI) quantifies acute poverty based on an aggregate index of health (malnutrition and child mortality), education, and standard of living. ${ }^{21}$ An MPI > 33\% classifies an individual as multi-dimensionally poor. Malnutrition was weighted higher because of lack of childhood mortality data. ${ }^{21}$ Malnutrition was defined as body mass index (BMI) < $18.5 \mathrm{~kg} / \mathrm{m}^{2}$ for subjects $>18$ years of age and as -2 SDs below the median BMI for age for those aged 6-18 years, according to the $\mathrm{WHO}^{22}$ Caste was categorized into scheduled caste (most disadvantaged), other backward caste (less disadvantaged than scheduled castes/tribes), and others. ${ }^{23}$ Maternal education was defined as the subject's mother having ever attended school. The facility where patients first sought care was categorized into private or public, as described elsewhere. ${ }^{24}$ Knowledge of TB transmission was defined as correctly identifying cough as the transmission mode, regardless of any additional modes reported. Diabetic patients were identified by self-report or random blood sugar level $\geq 200 \mathrm{mg} / \mathrm{dL}$.

Statistical analysis. Unadjusted and adjusted analyses were performed using logistic regression. Collinearity among variables was examined. Variable selection into the multivariable model was performed as follows. Variables with unadjusted $P<0.2$ were included along with age and at-risk alcohol use. Backward elimination removed variables in the order of decreasing $P$-value. Models with and without each removed variable in turn were compared to assess confounding, and the variable remained out of the model only if it did not substantially confound any of the other variables in the model. Interaction effects were assessed for biologically plausible terms. Symptoms at the 2-month follow-up visit were not considered because of substantial missing data among those with LTFU. Time from the beginning of the treatment until LTFU and month of LTFU were analyzed descriptively. Analyses were performed using SAS version 9.4 (SAS Institute Inc., Cary, NC).

Ethical approval and informed consent. The protocol was approved by the BMC (H-32657) and Rutgers (Pro2018002084) Institutional Review Boards, and the JIPMER Ethics and Scientific Advisory Committees (JIP/IEC/2013/4/194). All subjects provided informed consent in accordance with the Indian Council of Medical Research Ethical Guidelines for Biomedical Research on Human Participants and U.S. Code of Federal Regulations.

\section{RESULTS}

A total of 1,121 patients were registered from May 2014 through December 2017. Seventy-one patients met one of the following exclusion criteria: 40 had a household contact with MDR TB, 19 had 1 week of TB therapy or fluoroquinolone use, 11 had prior history of TB, and one planned to move away from the study area during treatment; 10 were excluded for meeting multiple exclusion criteria. Additionally, 46 patients were excluded for being critically ill. Of the remaining 994 patients enrolled into the study, LTFU was assessed for the 559 patients who were assigned a final treatment outcome. Of the 559 TB patients, 84 (15.0\%) were LTFU and 82/ $84(98 \%)$ of those LTFU were male. The remaining analyses are restricted to the $425 / 559$ (76.7\%) male TB patients (because nearly all LTFU occurred in males) and their 221 household contacts.

The mean age was slightly higher in the 82 males who were LTFU than the 343 males who were not ( 46.9 and 45.4 years, respectively; $P<0.001$; Table 1). At-risk alcohol use rates were higher in LTFU than in non-LTFU (56 [68.3\%] versus 173 [50.4\%]; $P=0.004$; Table 2), and those with at-risk alcohol use were more likely to be LTFU (odds ratio [OR] 2.20; 95\% Cl: 1.31-3.69).

In unadjusted analysis (Table 3), LTFU patients were more likely to be separated or divorced (OR 4.04; 95\% Cl: 1.51-10.83) or malnourished (OR 1.77; 95\% Cl: 1.03-3.05). Patients with diabetes were less likely to be LTFU (OR 0.50 ; 95\% Cl: 0.29-0.87). Age, average monthly income, knowledge that TB is transmitted by cough, and having a household contact who drinks alcohol or engages in at-risk alcohol use were not associated with LTFU.

In adjusted analyses (Table 3), being divorced/separated (aOR 3.80; 95\% Cl: 1.39-10.38) and at-risk alcohol use (aOR 1.92; $95 \% \mathrm{Cl}: 1.12-3.27)$ were significant predictors for 
TABLE 1

Sociodemographic characteristics of male TB patients who were lost to follow-up compared with males who successfully completed treatment in Puducherry and Tamil Nadu, India, from May 2014 to December $2017(n=425)$

\begin{tabular}{|c|c|c|c|c|}
\hline & LTFU, ${ }^{\star} N=82$ & Non-LTFU, ${ }^{\star} N=343$ & Total,$\uparrow N=425$ & $P$-valuloss to follow-up $\neq$ \\
\hline Age (years), mean (SD) & $46.9(11.7)$ & $45.4(13.9)$ & 45.7 (13.5) & $<0.001$ \\
\hline Marital status, $n(\%)$ & & & & 0.013 \\
\hline Married/living together & $63(19.5)$ & $260(80.5)$ & $323(76.8)$ & \\
\hline Never married & $7(10.8)$ & $58(89.2)$ & 65 (15.3) & \\
\hline Separated/divorced & $8(47.1)$ & 9 (52.9) & $17(4.0)$ & \\
\hline Widowed & $4(20.0)$ & $16(80.0)$ & $20(4.7)$ & \\
\hline Caste, $\S n(\%)$ & & & & 0.61 \\
\hline Scheduled caste/tribe & $24(21.2)$ & $89(78.8)$ & $113(26.6)$ & \\
\hline Other backward caste & 58 (18.9) & $249(81.1)$ & 307 (72.2) & \\
\hline Other & $0(0.0)$ & $5(100.0)$ & $5(1.2)$ & \\
\hline Religion, $n(\%)$ & & & & 0.18 \\
\hline Christianity & $5(19.2)$ & $21(80.8)$ & $26(6.1)$ & \\
\hline Hinduism & 76 (20.2) & $300(79.8)$ & 376 (88.5) & \\
\hline Islam & $1(4.4)$ & $22(95.7)$ & $23(5.4)$ & \\
\hline Location, $n(\%)$ & & & & $<0.001$ \\
\hline Puducherry & $39(14.1)$ & $238(85.9)$ & $277(65.2)$ & \\
\hline Tamil Nadu & $43(29.1)$ & 105 (70.9) & 148 (34.8) & \\
\hline Household monthly income, $n(\%)$ & & & & 0.065 \\
\hline$>\operatorname{Rs} 10,000(>\$ 148)$ & $6(9.8)$ & $55(90.2)$ & $61(14.4)$ & \\
\hline Rs $5,001-10,000(\$ 74-\$ 148)$ & $28(18.8)$ & $121(81.2)$ & $149(35.1)$ & \\
\hline Rs 3,000-5,000 (\$44-\$74) & $31(20.4)$ & $121(79.6)$ & $152(35.8)$ & \\
\hline$<\operatorname{Rs} 3,000(<\$ 44)$ & $17(30.9)$ & $38(69.1)$ & 55 (12.9) & \\
\hline Do not know & $0(0.0)$ & $7(100.0)$ & $7(1.7)$ & \\
\hline Refused to answer & $0(0.0)$ & $1(100.0)$ & $1(0.2)$ & \\
\hline Food insecurity, $\| n=422(\%)$ & & & & 0.18 \\
\hline Food secure & $64(17.8)$ & 296 (82.2) & $360(85.3)$ & \\
\hline Mildly food insecure & $7(35.0)$ & $13(65.0)$ & $20(4.7)$ & \\
\hline Moderately food insecure & $2(18.2)$ & $9(81.8)$ & $11(2.6)$ & \\
\hline Severely food insecure & $8(25.8)$ & $23(74.2)$ & $31(7.4)$ & \\
\hline Multi-dimensional poverty, $₫ n=422(\%)$ & & & & 0.11 \\
\hline Not poor & $20(14.7)$ & $116(85.3)$ & $136(32.2)$ & \\
\hline Poor & $61(21.3)$ & $225(78.7)$ & $286(67.8)$ & \\
\hline Maternal education, \# $n=400$ (\%) & & & & 0.11 \\
\hline None & $68(21.2)$ & $253(78.8)$ & $321(80.3)$ & \\
\hline Some & $10(12.7)$ & 69 (87.3) & $79(19.8)$ & \\
\hline
\end{tabular}

*Percentages are reported as row percentages.

$\dagger$ Percentages are reported as column percentage.

$\S$ Scheduled caste refers to the lowest caste whose members are among the most disadvantaged populations. Other backward caste is not only ranked above scheduled castes/tribes but also $\S$ Scheduled caste refers to the lowest
consists of disadvantaged population.

$\|$ Food insecurity was assessed by the household food insecurity access scale.

ๆ Multidimensional poverty was defined as having the MPI deprivation score $\geq 33 \%$.

\# Maternal education was defined as subject's mother having or never attended school.

increased risk of LTFU, and diabetes was a significant predictor for decreased risk of LTFU (aOR 0.52; 95\% Cl: 0.29--0.92). We excluded "municipality" from the final model, despite statistical significance in the unadjusted analysis, because we sought to uncover underlying reasons for a difference between municipalities that could be targeted for intervention.

There were 53 (65\%) LTFU with DOTS data on the date of last treatment dose. Among these, 23 (43\%) occurred within the first 2 months, and $43(81 \%)$ within the first 4 months; nine (17\%) occurred in August, and eight (15\%) in January (Figure 1).

\section{DISCUSSION}

This study aimed to identify predictors of LTFU among TB patients in southern India. In this disadvantaged patient population accessing care through RNTCP, there were high rates of at-risk alcohol use; understanding of TB curability and transmission was high. The LTFU rate was $19 \%$ among males, and $98 \%$ of LTFU was among males. Significant predictors of LTFU among males were at-risk alcohol use and being divorced or separated; having diabetes mellitus was protective against LTFU.
The LTFU percentage (15\% overall and 19\% among males) that we identified is three to four times higher than what has been reported for India overall $(4 \%)^{2}$ and for reports from the area of our study, Puducherry (4\%) and Tamil Nadu (5\%). ${ }^{2}$ Such high rates of LTFU have important implications for ongoing TB transmission in a country with more than a quarter of the TB cases worldwide. Rates of LTFU may in fact be higher than what we found, as we included only new patients, and LTFU rates are often higher for re-treatment cases. ${ }^{10,25}$

We found that at-risk alcohol use was associated with LTFU as has been found in many but not all previous studies. , 12,26,27 $^{2}$ Similarly at-risk alcohol use has been reported to affect HIV antiretroviral adherence in South India. ${ }^{28}$ Notably, having household contacts who drank or had at-risk alcohol use did not predict LTFU, suggesting household alcohol use does not drive adherence. Our findings suggest that in this region of India, high rates of at-risk alcohol use are driving outcomes and may subsequently impact transmission and development of drug resistance. ${ }^{5,6}$ Furthermore, because of the effect of alcohol use on initial engagement in care, continued efforts should be made to address alcohol consumption in TB patients. ${ }^{14,24}$ In the most recent publication of the National 
TABLE 2

Clinical and programmatic characteristics of male TB patients who were lost to follow-up compared with males who successfully completed treatment in Puducherry and Tamil Nadu, India, from May 2014 to December $2017(n=425)$

\begin{tabular}{|c|c|c|c|c|}
\hline & LTFU, ${ }^{\star} N=82$ & Non-LTFU, ${ }^{\star} N=343$ & Total,$\uparrow N=425$ & $P$-value $\neq$ \\
\hline Smoking, $n(\%)$ & & & & 0.041 \\
\hline Nonsmoker & $21(13.2)$ & $138(86.8)$ & 159 (37.4) & \\
\hline Former smoker & $35(22.4)$ & $121(77.6)$ & $156(36.7)$ & \\
\hline Current smoker & $26(23.6)$ & $84(76.4)$ & $110(25.9)$ & \\
\hline Drinking risk, $\S n(\%)$ & & & & 0.004 \\
\hline Not at risk & $26(13.3)$ & $170(86.7)$ & $196(46.1)$ & \\
\hline At risk & $56(24.5)$ & $173(75.5)$ & $229(53.9)$ & \\
\hline Malnutrition category, $\| n=422(\%)$ & & & & 0.17 \\
\hline Severely underweight & $28(23.5)$ & $91(76.5)$ & 119 (28.2) & \\
\hline Underweight & $32(21.5)$ & $117(78.5)$ & $149(35.3)$ & \\
\hline Normal & $19(13.6)$ & $121(86.4)$ & $140(33.2)$ & \\
\hline Overweight & $2(14.3)$ & $12(85.7)$ & $14(3.3)$ & \\
\hline Asthma, $n=310(\%)$ & & & & $>0.99$ \\
\hline No & $62(20.3)$ & $244(79.7)$ & $306(98.7)$ & \\
\hline Yes & $1(25.0)$ & $3(75.0)$ & $4(1.3)$ & \\
\hline Diabetes & & & & 0.010 \\
\hline No & $63(23.0)$ & $211(77.0)$ & $274(64.5)$ & \\
\hline Yes & $19(12.6)$ & $132(87.4)$ & $151(35.5)$ & \\
\hline $\mathrm{HIV}, n=423(\%)$ & & & & 0.35 \\
\hline Negative & $80(19.0)$ & $341(81.0)$ & $421(99.5)$ & \\
\hline Positive & $1(50.0)$ & $1(50.0)$ & $2(0.5)$ & \\
\hline Functional impairment, $₫ n(\%)$ & & & & 0.15 \\
\hline Normal & $22(15.2)$ & $123(84.8)$ & $145(34.1)$ & \\
\hline Unable to work & $60(21.4)$ & $220(78.6)$ & $280(65.9)$ & \\
\hline Facility of first care,\# $n=423(\%)$ & & & & 0.71 \\
\hline Private & $47(18.8)$ & $203(81.2)$ & $250(59.1)$ & \\
\hline Public & $35(20.2)$ & $138(79.8)$ & $173(40.9)$ & \\
\hline DOTS center, ${ }^{\star \star} n=330(\%)$ & & & & 0.70 \\
\hline $\mathrm{PHC}$ & $45(15.0)$ & $255(85.0)$ & $300(90.1)$ & \\
\hline Peripheral centers & $3(12.5)$ & $21(87.5)$ & $24(7.2)$ & \\
\hline $\mathrm{CHC}$ and TB clinics & $0(0.0)$ & $9(100.0)$ & $9(2.7)$ & \\
\hline Knowledge that TB is curable, $n(\%)$ & & & & 0.57 \\
\hline No & $5(23.8)$ & $16(76.2)$ & $21(4.9)$ & \\
\hline Yes & $77(19.1)$ & $327(80.9)$ & $404(95.1)$ & \\
\hline Knowledge that TB is transmitted by cough, $\dagger \dagger n(\%)$ & & & & 0.056 \\
\hline No & $25(26.3)$ & $70(73.7)$ & $95(22.4)$ & \\
\hline Yes & $57(17.3)$ & $273(82.7)$ & $330(77.7)$ & \\
\hline \multicolumn{5}{|l|}{ Symptoms at 2-month visit } \\
\hline 2 -month smear result, $n=305(\%)$ & & & & 0.61 \\
\hline Negative & $21(8.5)$ & $227(91.5)$ & $248(81.3)$ & \\
\hline Positive & $6(10.5)$ & $51(89.5)$ & $57(18.7)$ & \\
\hline Any symptoms, $n=304(\%)$ & & & & $>0.99$ \\
\hline No & $16(10.9)$ & $131(89.1)$ & $147(48.4)$ & \\
\hline Yes & $18(11.5)$ & $139(88.5)$ & $157(51.6)$ & \\
\hline Cough, $n=304(\%)$ & & & & 0.25 \\
\hline No & $19(9.5)$ & $180(90.5)$ & 199 (65.5) & \\
\hline Yes & $15(14.3)$ & $90(85.7)$ & $105(34.5)$ & \\
\hline Fever, $n=304(\%)$ & & & & 0.099 \\
\hline No & $28(10.1)$ & 249 (89.9) & $277(91.1)$ & \\
\hline Yes & $6(22.2)$ & $21(77.8)$ & $27(8.9)$ & \\
\hline Night sweat, $n=304$ (\%) & & & & $>0.99$ \\
\hline No & $32(94.1)$ & $253(93.7)$ & $285(93.8)$ & \\
\hline Yes & $2(5.9)$ & $17(6.3)$ & $19(6.3)$ & \\
\hline Weight loss, $n=293(\%)$ & & & & 0.41 \\
\hline No & $27(90.0)$ & $248(94.3)$ & $275(93.9)$ & \\
\hline Yes & $3(10.0)$ & $15(5.7)$ & $18(6.1)$ & \\
\hline \multicolumn{5}{|l|}{ Household contacts } \\
\hline Any household drinkers, $n=221(\%)$ & & & & 0.20 \\
\hline No & $26(68.4)$ & $144(78.7)$ & $170(76.9)$ & \\
\hline Yes & $12(31.6)$ & $39(21.3)$ & $51(23.1)$ & \\
\hline Any at-risk household drinkers, $n=220(\%)$ & & & & 0.16 \\
\hline No & $33(86.8)$ & $172(94.0)$ & 205 (92.8) & \\
\hline Yes & $5(13.2)$ & $11(6.0)$ & $16(7.2)$ & \\
\hline
\end{tabular}

DOTS = directly observed therapy, short course

Percentages are reported as row percentages.

$+P$-values from Fisher's exact tests for categorical variables, and $t$-tests for age

§ Drinking risk was assessed by Alcohol Use Disorders Identification Test (AUDIT-C) score $\geq 3$ for females and $\geq 4$ for males. $\|$ Malnutrition categories were defined as follows: severely underweight $(\mathrm{BMI} \leq 16 \mathrm{~kg} / \mathrm{m} 2)$, underweight $\left(16<\mathrm{BMl} \leq 18.5 \mathrm{~kg} / \mathrm{m}^{2}\right)$, normal $\left(18.5<\mathrm{BMl}<25 \mathrm{~kg} / \mathrm{m}^{2}\right)$, and overweight $(25 \leq \mathrm{BMl}<30$

I Functional impairment was assessed by the Karnofsky performance score (KPS) $\leq 70$.

\# Facility where patient first sought care were categorized as private or public institutions. Private facilities include pharmacies, private allopathic clinics, medical college hospitals, and nonallopathic clinics. Public facilities included government hospitals, primary health centers (PHCs), and municipal corporation hospitals.

** DOTS centers included primary health center (PHC), Hemericx center (HRC), district tuberculosis center (DTC), community health center (CHC), clinics in Anganwadi, and subcenters. HRC and DTC were grouped together as peripheral centers. CHC, Anganwadi clinics, and subcenters were grouped as CHC and TB clinics.

†† Knowledge of TB transmission was defined for patients who correctly identified coughing as the transmission mode, regardless of any additional modes reported. 
TABLE 3

Results of the unadjusted and adjusted analyses of loss to follow-up among male TB patients in Puducherry and Tamil Nadu, India ( $n=422)$

\begin{tabular}{|c|c|c|}
\hline & Unadjusted ( $N=422)$ OR $(95 \% \mathrm{Cl})$ & Adjusted $(N=422)$ OR $(95 \% \mathrm{Cl}$ \\
\hline Age (increase of 1 year) & $1.01(0.99-1.03)$ & $1.01(0.99-1.03)$ \\
\hline \multicolumn{3}{|l|}{ Marital status } \\
\hline Married/single/widowed & Reference & Reference \\
\hline Separated/divorced & $4.04(1.51-10.83)$ & $3.80(1.39-10.38)$ \\
\hline \multicolumn{3}{|l|}{ Religion } \\
\hline Hinduism & Reference & - \\
\hline Christianity/Islam & $0.57(0.23-1.39)$ & - \\
\hline \multicolumn{3}{|l|}{ Household monthly income } \\
\hline$\leq \operatorname{Rs} 5,000(\$ 74)$ & Reference & - \\
\hline$>\operatorname{Rs} 5,000(\$ 74)$ & $1.53(0.94-2.50)$ & - \\
\hline \multicolumn{3}{|l|}{ Smoking } \\
\hline Nonsmoker & Reference & - \\
\hline Former/current smoker & $1.92(1.12-3.30)$ & - \\
\hline \multicolumn{3}{|l|}{ Alcohol use ${ }^{*}$} \\
\hline Not at risk & Reference & Reference \\
\hline At risk & $2.20(1.31-3.69)$ & $1.92(1.12-3.27)$ \\
\hline \multicolumn{3}{|l|}{ Malnutrition category $\dagger$} \\
\hline Normal/overweight & Reference & - \\
\hline Underweight/severely underweight & $1.77(1.03-3.05)$ & - \\
\hline \multicolumn{3}{|l|}{ Diabetes } \\
\hline No & Reference & Reference \\
\hline Yes & $0.50(0.29-0.87)$ & $0.52(0.29-0.92)$ \\
\hline \multicolumn{3}{|c|}{ Knowledge that TB is transmitted by cough $\ddagger$} \\
\hline No & Reference & - \\
\hline Yes & $0.61(0.36-1.06)$ & - \\
\hline
\end{tabular}

Family Health Survey India, alcohol consumption among men was reported to be $29.2 \%$ nationally and upward of $40 \%$ in the districts of Puducherry and Tamil Nadu. ${ }^{29}$ Given the high prevalence of alcohol consumption, this is a particularly relevant predictor of LTFU. One component of this approach might be a collaborative framework for TB that addresses not only alcohol consumption but also tobacco use and diabetes. ${ }^{30,31}$

Notably, those who were divorced or separated also were more likely to be LTFU. Social support has been found to be a predictor of adherence in studies of HIV in India. ${ }^{32}$ Recently, social support has also been cited in lowering stigmatization and improving adherence of anti-TB treatment. ${ }^{33,34}$ Individuals at risk for LTFU (or who start to miss DOTS visits) could benefit from more frequent follow-up by community health workers.

Patients with diabetes were less likely to be LTFU, which is the first time that this has been reported to our knowledge. The findings are in contrast to previously published data showing no differences in LTFU rates in Saudi Arabia and India in diabetic and non-diabetic patients, and unadjusted analyses from China showing increased LTFU in diabetics with TB; these studies all had low LTFU rates. ${ }^{32,35-37}$ It is possible that in South India, those diagnosed with diabetes were more familiar with the healthcare system than non-diabetics, and this awareness facilitated adherence to treatment. Increased interactions with healthcare providers are opportunities for education about medical conditions; such knowledge may improve adherence. There may be lessons that can be extrapolated from this population to non-diabetic TB patients. It is also possible that TB in diabetics was detected earlier (as the $\mathrm{WHO}$ and RNTCP have emphasized routine bidirectional screening) and this earlier diagnosis facilitated improved outcomes or that diabetics had fewer adverse drug reactions (and hence were more adherent to medication) because of lower drug levels, although data are conflicting. ${ }^{38-40}$ Conversely, it is possible that patients with diabetes are less likely to be LTFU as they perceive themselves at increased risk of poor outcomes. Nonetheless, it is clear that the two epidemics of diabetes mellitus and TB are linked; diabetes mellitus is a risk factor for TB disease and associated with increased rates of TB treatment failure, death, and impaired mycobacterial clearance. ${ }^{14,30,36,41,42}$ This finding is particularly relevant given the prevalence of diabetes mellitus in India (74 million adults or $8.9 \%$ of the population) and the expected doubling in prevalence by $2045 .{ }^{43}$ Closely linked diabetes and TB treatment programs will be critical to TB elimination in India and elsewhere.

Nearly half of the men were LTFU within the first 2 months of treatment and $80 \%$ within the first 4 months, consistent with previous studies. ${ }^{10,12}$ Early treatment cessation may reflect improvement in symptoms and/or patients' beliefs that they have been cured. Whereas length of time until LTFU has been reported in prior studies, the seasonality of LTFU has not previously been explored. In our study, a large proportion of men were lost to follow-up in August and September, which coincides with monsoon season, when South India receives most of its annual precipitation. ${ }^{44}$ Poor weather conditions may adversely affect patients' ability to commute to DOTS centers and impact treatment adherence; healthcare worker monitoring of patients could also be hampered by heavy rain. The large proportion of LTFU in January (when Pongal and other major holidays are celebrated) is consistent with the observation that treatment-seeking behavior is affected during major festivals. The small number of events prevents making definitive conclusions regarding timing.

The strength of this study is our ability to evaluate wellcharacterized epidemiologic predictors of LTFU (including at-risk alcohol use using AUDIT-C and TB knowledge using validated questionnaires). Furthermore, we collected data for a large cohort in an area for which there were minimal data on LTFU 


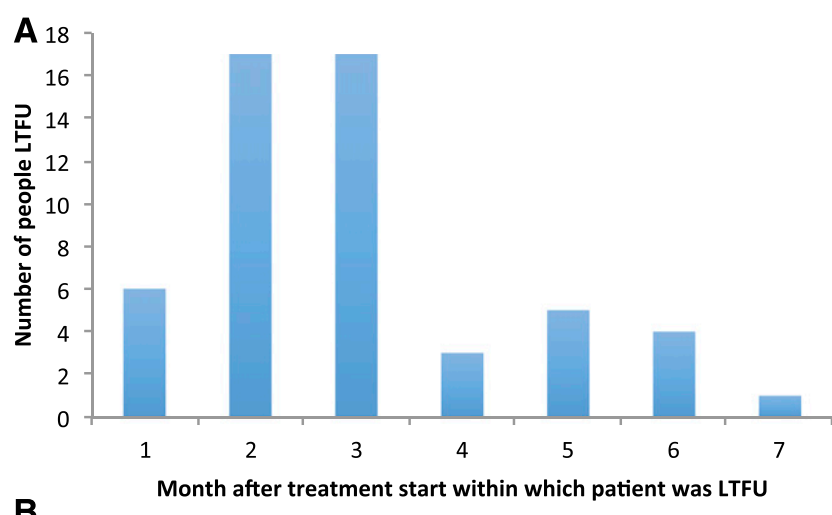

B

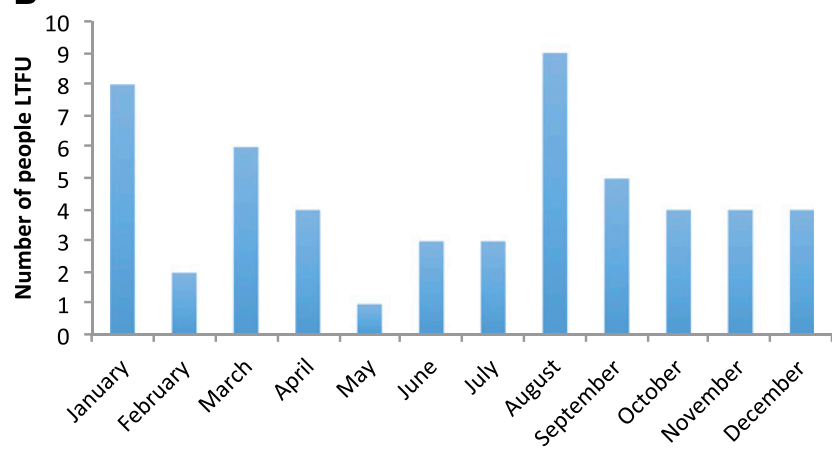

Calendar month of LTFU

FIGURE 1. The number of patients lost to follow-up for the 53 LTFU patients with directly observed therapy, short course data on date of last treatment dose by $(\mathbf{A})$ the length of time from the start of the treatment until LTFU. (B) The month of the year. This figure appears in color at www.ajtmh.org.

predictors. Our focus on individuals accessing care through RNTCP limits application of our findings to those treated in private clinics, and the predictors of LTFU identified for men may not apply to women; however, in this region, it is clear that most incidences of LTFU occur in men. Limitations such as exclusion of critically ill patients from the study suggest that our results are not applicable to this particular subgroup. Furthermore, use of random blood glucose may not identify all diabetic patients, and in some instances, the diagnosis of diabetes was made by patient self-report, which may bias the effect of diabetes as a risk factor of LTFU. The prevalence of risk factors among this subpopulation of TB patients may also affect the generalizability of the results. Another limitation is our reliance on RNTCP documentation. DOTS treatment records were not completed or unavailable for many subjects (particularly for those who had already completed treatment), and treatment adherence was not independently verified other than through participant self-report. Because of incomplete timing data, time-to-event analyses were not performed. Programmatic efforts are needed to improve access to documentation and ensure complete records.

\section{CONCLUSION}

The high rate of LTFU in our study in southern India has critical implications for TB treatment program success. The increased odds of LTFU in male patients with at-risk alcohol use and those who are divorced/separated suggest that TB programs may benefit from interventions based on more intensive follow-up and more frequent visits from healthcare workers. Exploring factors that have enabled improved adherence among diabetic TB patients may help improve adherence rates overall; increased contact with the healthcare system may play a role for these patients. As India aims to eliminate TB by $2025{ }^{2}$ identifying local factors that impact care will be particularly important in this large, culturally heterogeneous country.

Received May 30, 2019. Accepted for publication March 18, 2020.

Published online July 6, 2020.

Acknowledgments: We would like to thank all of the participants and their families without whom the study would not have been possible. We are also grateful to the study teams who have worked tirelessly for years and to Laura White for her review of the manuscript.

Financial support: This project has been funded by award no. USB$31150-X X-13$ of the U.S. Civilian Research and Development Foundation (CRDF Global) and by the National Science Foundation under cooperative agreement no. OISE-9531011 with federal funds from the government of India's (GOI) Department of Biotechnology (DBT), the Indian Council of Medical Research (ICMR), the U.S. National Institutes of Health (NIH), National Institute of Allergy and Infectious Diseases (NIAID), and Office of AIDS Research (OAR), and distributed in part by CRDF Global.

Disclaimer: The contents of this publication are solely the responsibility of the authors and do not represent the official views of the DBT, the ICMR, the NIH, or CRDF Global. TJZ and HEJ were supported by the NIH (NIH-GMS T32GM074905 and U.S. NIH K01Al102944 awards, respectively).

Authors' addresses: Thomas J. Zhou and Helen E. Jenkins, Department of Biostatistics, Boston University School of Public Health, Boston, MA, E-mails: tjzhou@bu.edu and helenje@bu.edu. Subitha Lakshminarayanan, Sonali Sarkar, and Gautam Roy, Department of Preventive and Social Medicine, Jawaharlal Institute of Postgraduate Medical Education and Research, Puducherry, India, E-mails: subitha.l@gmail.com, sarkarsonaligh@gmail.com, and gautam.r@ jipmer.edu.in. Selby Knudsen, Department of Medicine, Section of Infectious Diseases, Boston University School of Medicine, Boston, MA, E-mail: selby.knudsen@bmc.org. C. Robert Horsburgh Jr., Department of Biostatistics, Boston University School of Public Health, Boston, MA, Department of Medicine, Section of Infectious Diseases, Boston University School of Medicine, Boston, MA, and Department of Epidemiology, Boston University School of Public Health, Boston, MA, E-mail: rhorsbu@bu.edu. Muthuraj Muthaiah, Intermediate Reference Laboratory, Government Hospital for Chest Diseases, Puducherry, India, E-mail: muthuraj1970@gmail.com. Carolyn K. Kan, Department of Medicine, Boston University School of Medicine, Boston, MA, E-mail: ckan2@bu.edu. Padmini Salgame and Jerrold J. Ellner, Department of Medicine, Rutgers New Jersey Medical School, Newark, NJ, E-mails: salgampa@njms.rutgers.edu and ellnerjj@njms.rutgers.edu. Natasha S. Hochberg, Department of Medicine, Section of Infectious Diseases, Boston University School of Medicine, Boston, MA, and Department of Epidemiology, Boston University School of Public Health, Boston, MA, E-mail: nhoch@bu.edu.

This is an open-access article distributed under the terms of the Creative Commons Attribution (CC-BY) License, which permits unrestricted use, distribution, and reproduction in any medium, provided the original author and source are credited.

\section{REFERENCES}

1. World Health Organization, 2019. Global Tuberculosis Report 2019. Geneva, Switzerland: WHO.

2. Central TB Division, Directorate General of Health Services, Ministry of Health with Family Welfare, Nirman Bhavan, 2018. India TB Report 2018. Revised National Tuberculosis Control Programme Annual Status Report. New Delhi, India: Ministry of Health and Family Welfare.

3. Reid MJA et al., 2019. The lancet commissions building a tuberculosis-free world: the lancet commission on tuberculosis. Lancet 393: 1331-1384. 
4. Kolappan C, Subramani R, Karunakaran K, Narayanan P, 2006. Mortality of tuberculosis patients in Chennai, India. Bull World Health Organ 84: 555-560.

5. Verver S, Warren RM, Beyers N, Richardson M, van der Spuy GD, Borgdorff MW, Enarson DA, Behr MA, van Helden PD, 2005. Rate of reinfection tuberculosis after successful treatment is higher than rate of new tuberculosis. Am J Respir Crit Care Med 171: 1430-1435.

6. Pablos-Mendez A, Knirsch CA, Barr RG, Lerner BH, Frieden TR, 1997. Nonadherence in tuberculosis treatment: predictors and consequences in New York city. Am J Med 102: 164-170.

7. Saqib SE, Ahmad MM, Amezcua-Prieto C, Virginia M-R, 2018. Treatment delay among pulmonary tuberculosis patients within the Pakistan national tuberculosis control program. Am J Trop Med Hyg 99: 143-149.

8. Jaggarajamma K, Sudha G, Chandrasekaran V, Nirupa C, Thomas A, Santha T, Muniyandi M, Narayanan P, 2007. Reasons for non-compliance among patients treated under revised national tuberculosis control programme (RNTCP), tiruvallur district, south India. Indian J Tuberc 54: 130-135.

9. Santha T et al., 2002. Risk factors associated with default, failure and death among tuberculosis patients treated in a DOTS programme in Tiruvallur district, south India, 2000. Int J Tuberc Lung Dis 6: 780-788.

10. Babiarz KS, Suen S, Goldhaber-Fiebert JD, 2014. Tuberculosis treatment discontinuation and symptom persistence: an observational study of Bihar, India's public care system covering $>100,000,000$ inhabitants. BMC Public Health 14: 418.

11. Jaiswal A, Singh V, Ogden J, Porter J, Sharma P, Sarin R, Arora V, Jain R, 2003. Adherence to tuberculosis treatment: lessons from the urban settings of Delhi, India. Trop Med Int Health 8: 625-633.

12. Roy N, Basu M, Das S, Mandal A, Dutt D, Dasgupta S, 2015. Risk factors associated with default among tuberculosis patients in Darjeeling district of West Bengal, India. J Fam Med Prim Care 4: 388-394.

13. Mehra D, Kaushik RM, Kaushik R, Rawat J, Kakkar R, 2013. Initial default among sputum-positive pulmonary TB patients at a referral hospital in Uttarakhand, India. Trans $R$ Soc Trop Med Hyg 107: 558-565.

14. Hochberg NS et al., 2017. Comorbidities in pulmonary tuberculosis cases in Puducherry and Tamil Nadu, India: opportunities for intervention. PLoS One 12: e0183195.

15. Leong $S$ et al., 2018. Existing blood transcriptional classifiers accurately discriminate active tuberculosis from latent infection in individuals from south India. Tuberculosis (Edinb) 109: 41-51.

16. Kubiak RW et al., 2019. Interaction of nutritional status and diabetes on active and latent tuberculosis: a cross-sectional analysis. BMC Infect Dis 19: 1-9.

17. Hoyt KJ et al., 2019. Effect of malnutrition on radiographic findings and mycobacterial burden in pulmonary tuberculosis. PLOS One 14: 1-11.

18. Central TB Division, Directorate General of Health Services, Ministry of Health with Family Welfare, Nirman Bhavan, 2017. National Strategic Plan for Tuberculosis Elimination 2017-2025. Revised National Tuberculosis Control Programme. New Delhi, India: Ministry of Health and Family Welfare.

19. Bush K, Kivlahan DR, McDonell MB, Finn SD, Bradley KA, 1998. The AUDIT alcohol consumption questions (AUDIT-C): an effective brief screening test for problem drinking. Arch Intern Med 158: 1789-1795.

20. Coates J, Swindale A, Bilinsky P, 2007. Household Food Insecurity Access Scale (HFIAS) for Measurement of Household Food Access: Indicator Guide (v.3). Washington, DC: FHI 360/ FANTA.

21. Alkire S, Santos ME, 2014. Measuring acute poverty in the developing world: robustness and scope of the multidimensional poverty index. World Dev 59: 251-274.

22. World Health Organization, 2018. BMI Classification. Geneva, Switzerland: WHO.

23. Suryanarayana $M H, 2001$. Scheduled castes, their disadvantages and deprivation: a rural all-India profile. Artha Vijnana 43: 84-97.
24. Van Ness SE et al., 2017. Predictors of delayed care seeking for tuberculosis in southern India: an observational study. BMC Infect Dis 17: 567.

25. Vasudevan K, Jayakumar N, Gnanasekaran D, 2014. Smear conversion, treatment outcomes and the time of default in registered tuberculosis patients on RNTCP DOTS in Puducherry, south India. J Clin Diagn Res 8: JC05-JC08.

26. Chang KC, Leung CC, Tam CM, 2004. Risk factors for defaulting from anti-tuberculosis treatment under directly observed treatment in Hong Kong. Int J Tuberc Lung Dis 8: 1492-1498.

27. Vijay S, Kumar P, Chauhan LS, Vollepore BH, Kizhakkethil UP, Rao SG, 2010. Risk factors associated with default among new smear positive TB patients treated under DOTS in India. PLoS One 5: e10043.

28. Yathiraj AB, Unnikrishnan B, Ramapuram JT, Kumar N, Mithra P, Kulkarni V, Holla R, Darshan B, Thapar R, 2016. Factors influencing adherence to antiretroviral therapy among people living with HIV in coastal south India. $J$ Int Assoc Provid AIDS Care 15: 529-533.

29. International Institute for Population Sciences (IIPS) and ICF, 2017. National Family Health Survey (NFHS-4), 2015-2016. Mumbai, India: Ministry of Health and Family Welfare.

30. Lönnroth K, Roglic G, Harries A, 2014. Improving tuberculosis prevention and care through addressing the global diabetes epidemic: from evidence to policy and practice. Lancet Diabetes Endocrinol 2: 730-739.

31. Pizzol D, Di Gennaro F, Chhaganlal KD, Fabrizio C, Monno L, Putoto G, Saracino A, 2016. Tuberculosis and diabetes: current state and future perspectives. Trop Med Int Health 21: 694-702.

32. Kumarasamy N, Safren SA, Raminani SR, Pickard R, James R, Krishnan AK, Solomon S, Mayer KH, 2005. Barriers and facilitators to antiretroviral medication adherence among patients with HIV in Chennai, India: a qualitative study. AIDS Patient Care STDS 19: 526-537.

33. Yan S, Zhang S, Tong Y, Yin X, Lu Z, Gong Y, 2018. Nonadherence to antituberculosis medications: the impact of stigma and depressive symptoms. Am J Trop Med Hyg 98: 262-265.

34. Daftary A, Mitchell EMH, Reid MJA, Fekadu E, Goosby E, 2018. To end TB, first-ever high-level meeting on tuberculosis must address stigma. Am J Trop Med Hyg 99: 1114-1116.

35. Singla R, Khan N, Al-Sharif N, Al-Sayegh MO, Shaikh MA, Osman MM, 2006. Influence of diabetes on manifestations and treatment outcome of pulmonary TB patients. Int J Tuberc Lung Dis 10: 74-79.

36. Viswanathan V, Vigneswari A, Selvan K, Satyavani K, Rajeswari R, Kapur A, 2014. Effect of diabetes on treatment outcome of smear-positive pulmonary tuberculosis-a report from south India. J Diabetes Complications 28: 162-165.

37. Mi F et al., 2013. Diabetes mellitus and tuberculosis: pattern of tuberculosis, two-month smear conversion and treatment outcomes in Guangzhou, China. Trop Med Int Health 18: 1379-1385.

38. Requena-Méndez A, Davies G, Ardrey A, Jave O, López-Romero SL, Ward SA, Moore DAJ, 2012. Pharmacokinetics of rifampin in Peruvian tuberculosis patients with and without comorbid diabetes or HIV. Antimicrob Agents Chemother 56: 2357-2363.

39. Martinez N, Kornfeld H, 2014. Diabetes and immunity to tuberculosis. Eur J Immunol 44: 617-626.

40. Nijland HMJ, Ruslami R, Stalenhoef JE, Nelwan EJ, Alisjahbana B, Nelwan $\mathrm{RHH}$, van der Ven AJAM, Danusantoso H, Aarnoutse $\mathrm{RE}$, Crevel RVan, 2006. Exposure to rifampicin is strongly reduced in patients with tuberculosis and type 2 diabetes. Clin Infect Dis 43: 848-854.

41. Baker MA, Lin $\mathrm{H}$, Chang $\mathrm{H}$, Murray MB, 2012. The risk of tuberculosis disease among persons with diabetes mellitus: a prospective cohort study. Clin Infect Dis 54: 818-825.

42. Kornfeld H, West K, Kane K, Kumpatla S, Zacharias RR, MartinezBalzano C, Li W, Viswanathan V, 2016. High prevalence and heterogeneity of diabetes in patients with TB in south India. Chest 149: 1501-1508.

43. International Diabetes Federation, 2019. IDF Diabetes Atlas, 9th edition. Brussels, Belgium: International Diabetes Federation.

44. World Bank Group, 2019. Climate Data: India. Available at: https:// climateknowledgeportal.worldbank.org/country/india/climatedata-historical. Accessed May 7, 2019. 